Supplement of Geosci. Model Dev., 12, 849-878, 2019

https://doi.org/10.5194/gmd-12-849-2019-supplement

(C) Author(s) 2019. This work is distributed under

the Creative Commons Attribution 4.0 License.

(c) (1)

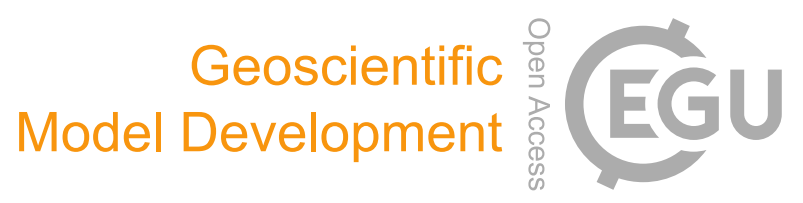

Supplement of

\title{
Mechanistic representation of soil nitrogen emissions in the Community Multiscale Air Quality (CMAQ) model v 5.1
}

Quazi Z. Rasool et al.

Correspondence to: Quazi Z. Rasool (qzr1@email.unc.edu)

The copyright of individual parts of the supplement might differ from the CC BY 4.0 License. 
Figure S1 Percentage contribution of soil $\mathrm{NO}+\mathrm{HONO}$ to Total $\mathrm{NO}_{x}(\mathrm{NO}+$ $\mathrm{NO}_{2}$ ) + HONO emissions on a monthly average basis for May (left) and July (right) 2011 for: a) YL scheme, b) Parametrized BDSNP scheme and c) Mechanistic scheme. Note: only the mechanistic scheme estimates HONO in addition to NO

a)
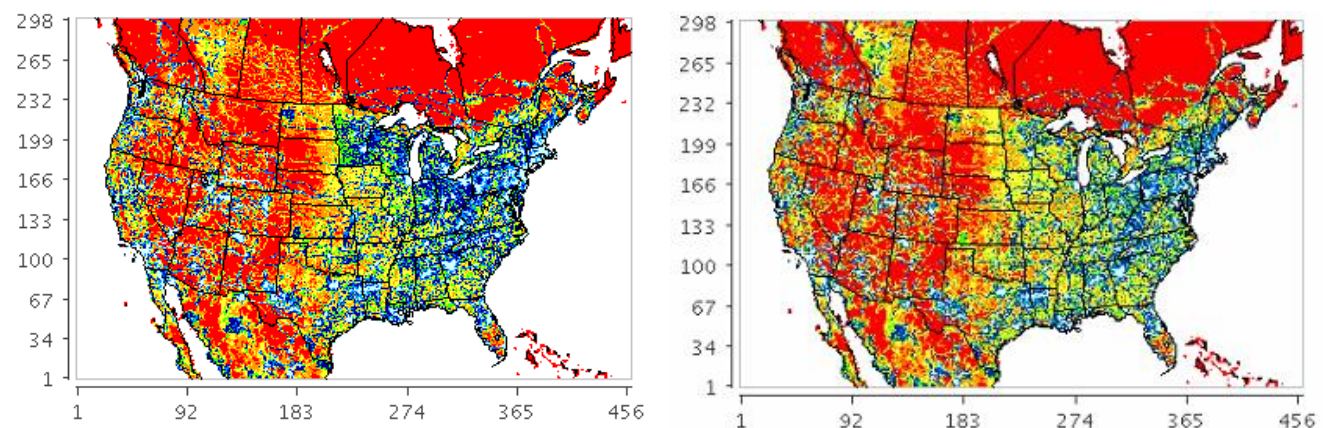

b)
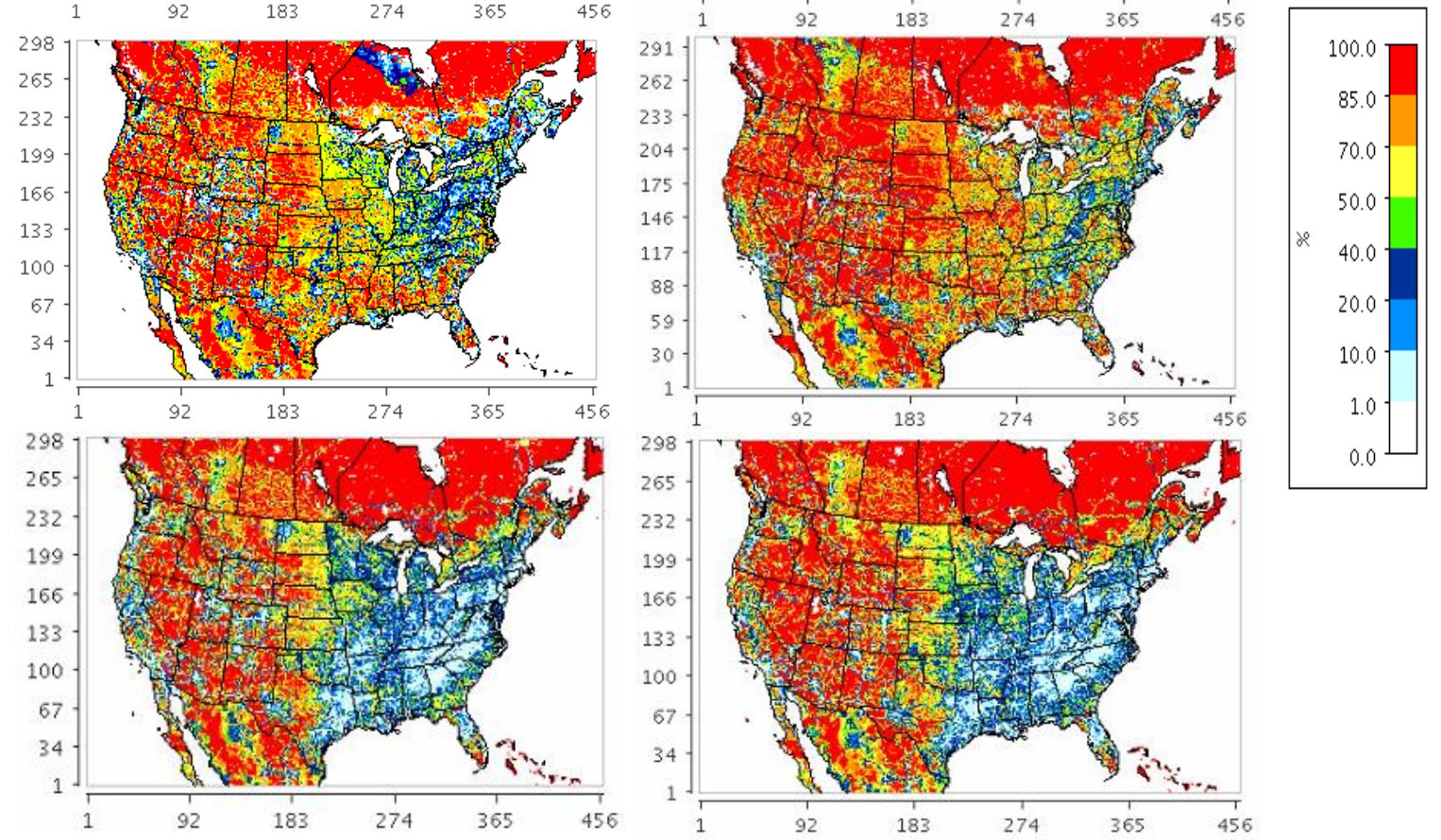

c)

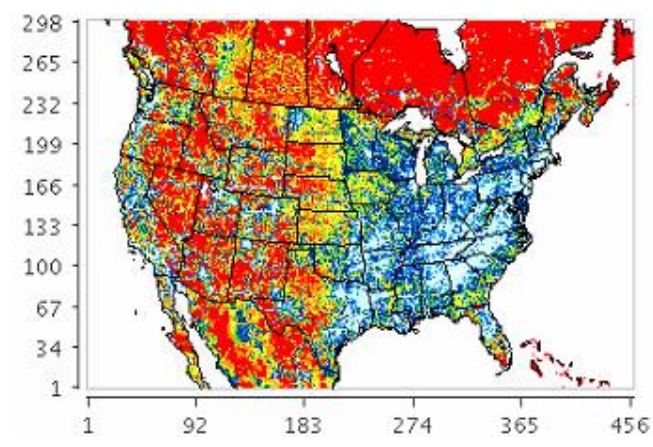


Figure S2 Comparison of average monthly (May 2011) Mean Bias (MB) for $\mathrm{CMAQ} \mathrm{O}_{3}(\mathrm{ppb})$ using $\mathrm{YL}(\mathrm{a})$ and Mechanistic schemes (b) evaluated against $A Q \mathrm{O}_{3}$ observations
(a) Ozone MB (YL - AQS)
(b) Ozone MB (Mech. - AQS)

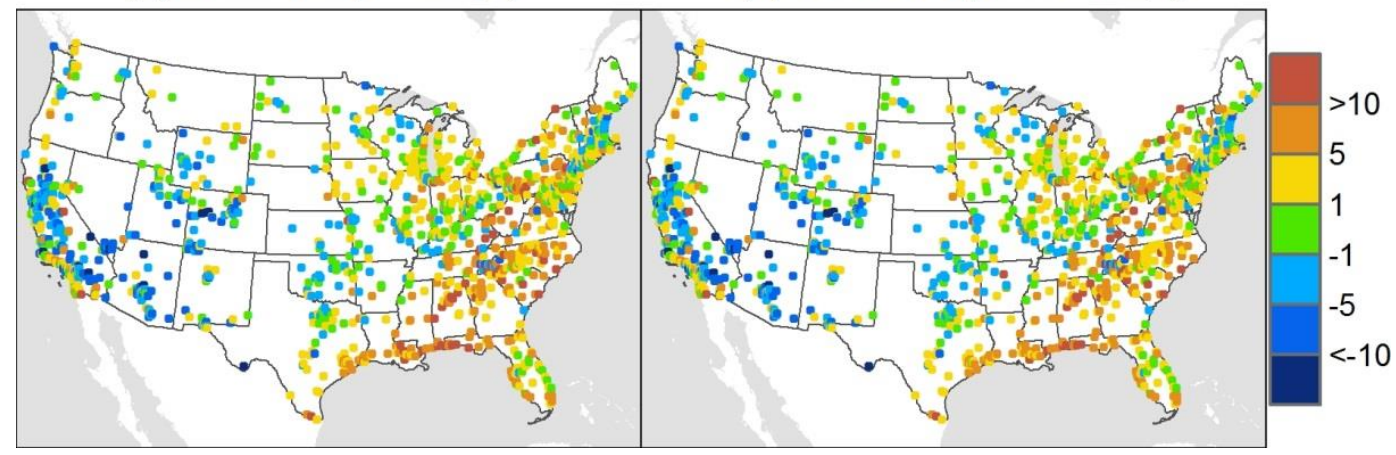

Figure S3 Comparison of average monthly (May2011) MB for CMAQ NO (NO $+\mathrm{NO}_{2}$ ) (ppb) using $\mathrm{YL}$ (a) and Mechanistic schemes (b) evaluated against AQS $\mathrm{NO}_{x}$ observations

(a) May NOx MB (YL - AQS) (b) May NOx MB (Mech. - AQS)

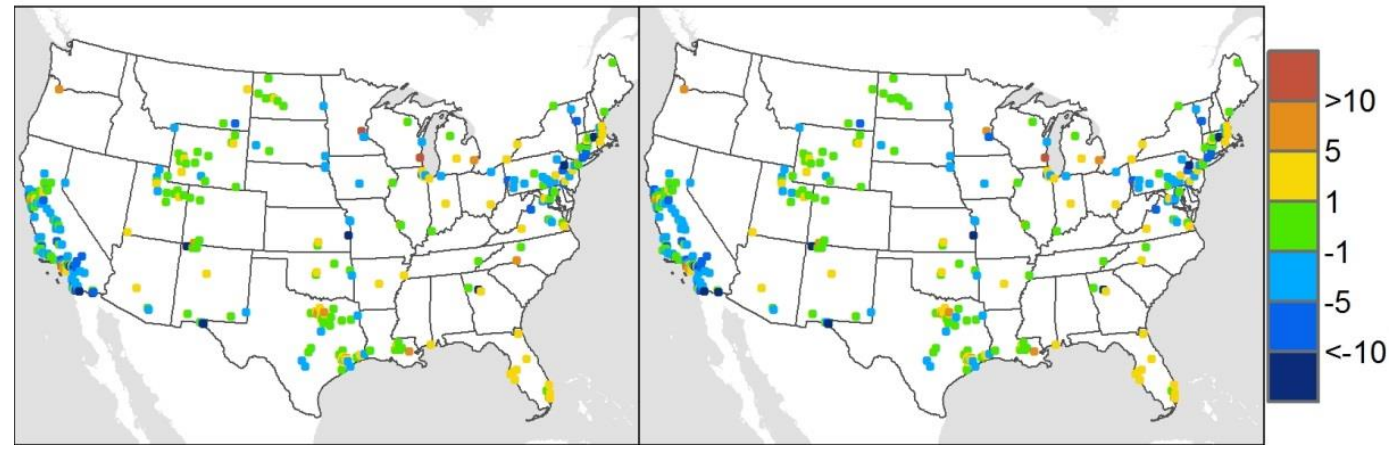


Figure S4 Comparison of average monthly (May 2011) MB for $\mathrm{CMAQ} \mathrm{O}_{3}$ (ppb) using YL (a) and Mechanistic schemes (b) evaluated against CASTNET $\mathrm{O}_{3}$ observations

(a) Ozone MB (YL - CASTNET)

(b) Ozone MB (Mech. - CASTNET)

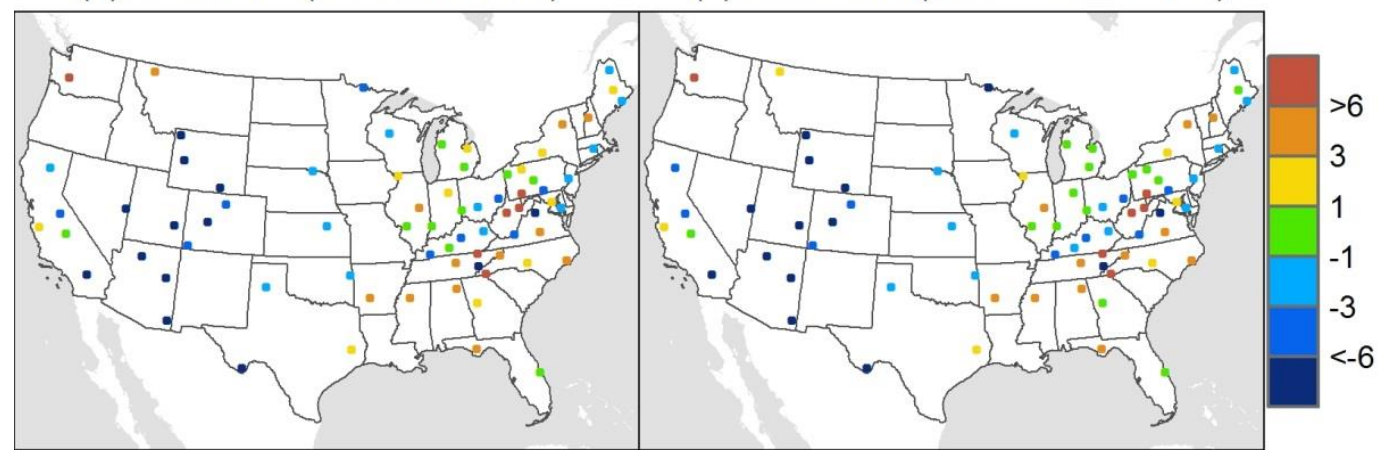

Figure S5 Comparison of average monthly (May 2011) MB for CMAQ PM2.5 $\mathrm{NO}_{3}\left(\mu \mathrm{g} / \mathrm{m}^{3}\right)$ using $\mathrm{YL}(\mathrm{a})$ and Mechanistic schemes (b) evaluated against IMPROVE $\mathrm{PM}_{2.5} \mathrm{NO}_{3}$ observations

$\begin{array}{ll}\text { (a) PM25 NO3 MB (YL-IMPROVE) } & \text { (b) PM25 NO3 MB (Mech.-IMPROVE) }\end{array}$

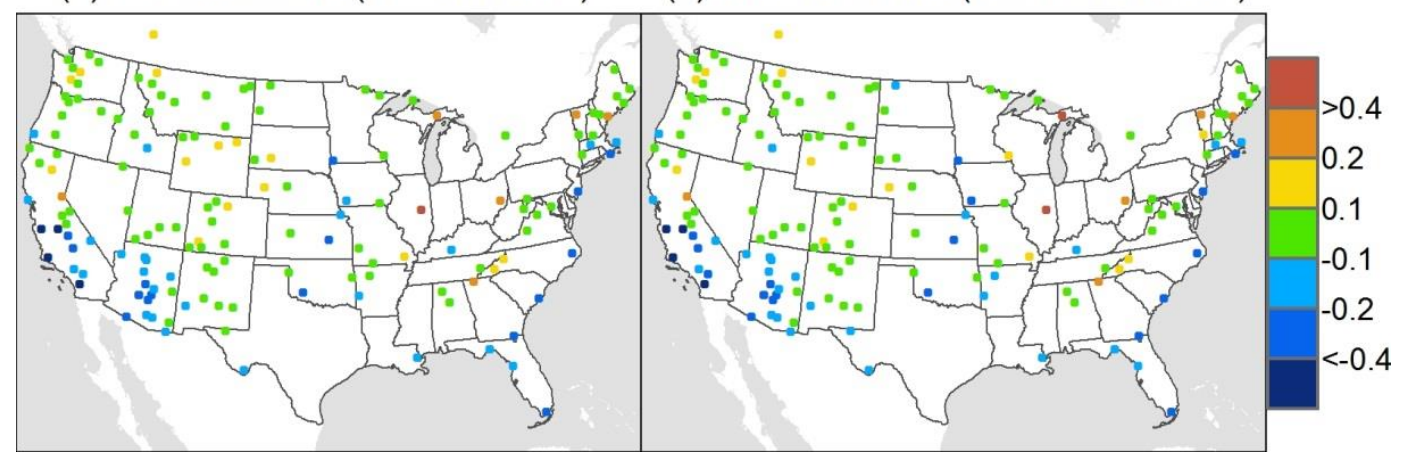


Figure S6 Comparison of average monthly (July 2011) MB for CMAQ PM 2.5 $\mathrm{NO}_{3}\left(\mu \mathrm{g} / \mathrm{m}^{3}\right)$ using $\mathrm{YL}(\mathrm{a})$ and Mechanistic schemes (b) evaluated against CSN $\mathrm{PM}_{2.5} \mathrm{NO}_{3}$ observations
(a) PM25 NO3 NMB (YL - CSN)
(b) PM25 NO3 NMB (Mech. - CSN)

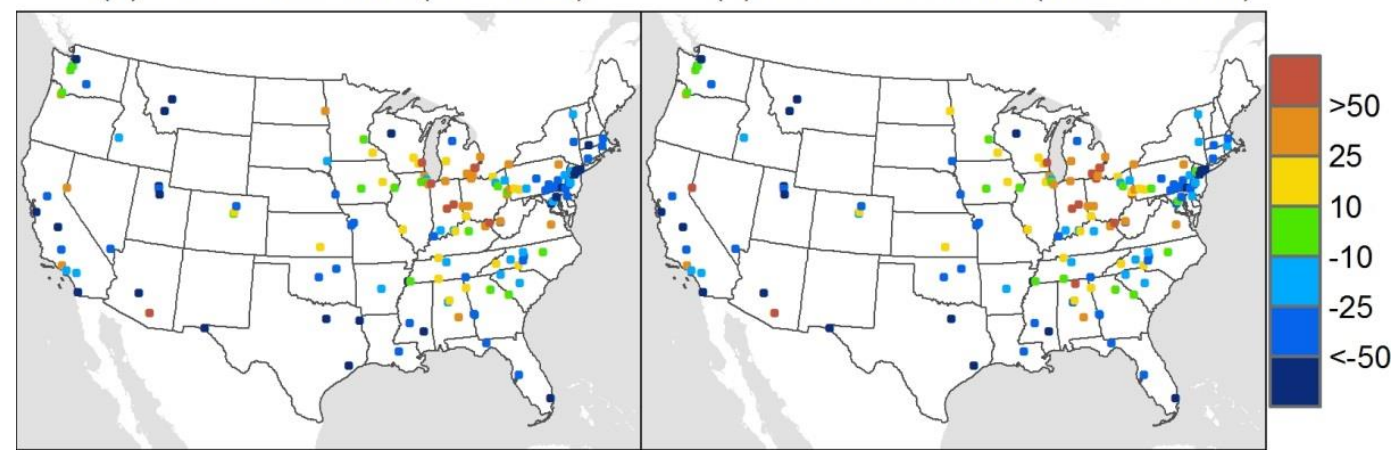


Figure $\mathrm{S} 7$ Soil moisture $\left(\mathrm{m}^{3} / \mathrm{m}^{3}\right)$ on a monthly mean basis (May and July 2011) over the modeling domain

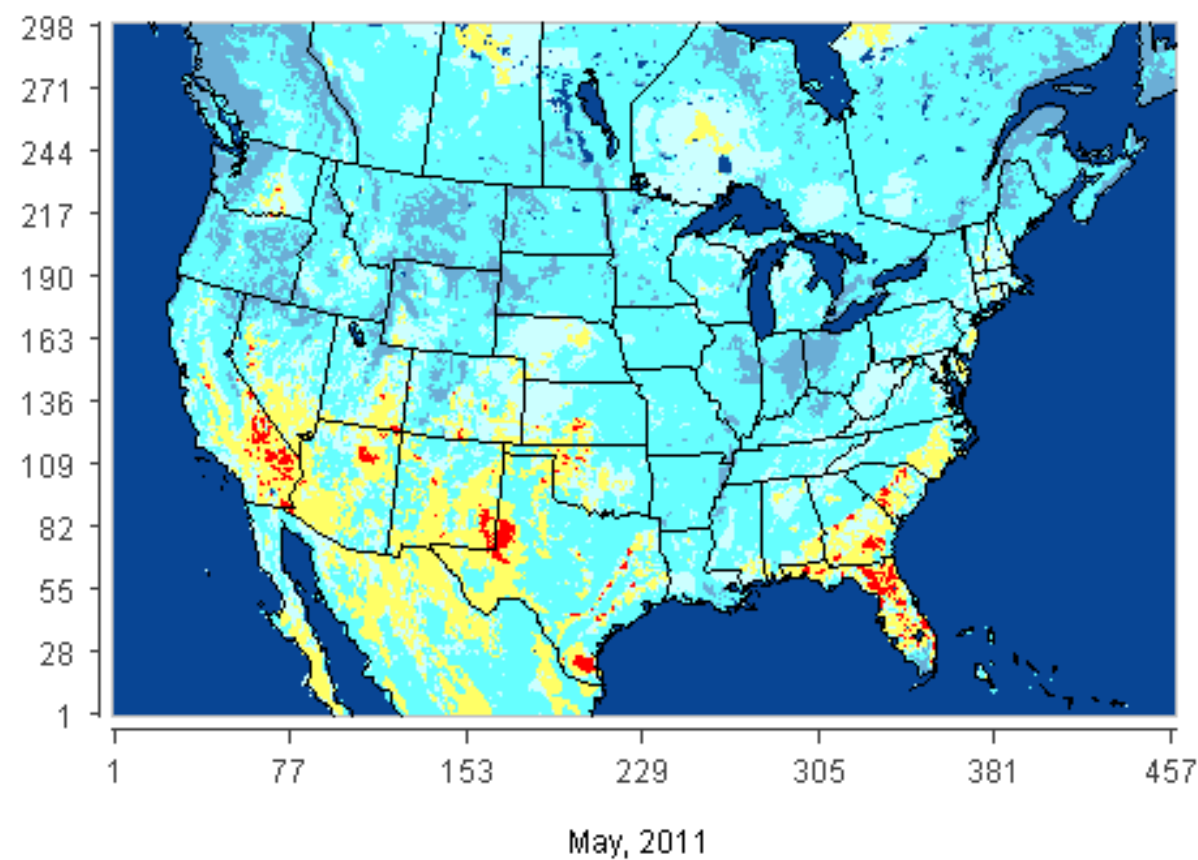

$\operatorname{Min}(69,128)=0.05, \operatorname{Max}(2,1)=1.00$
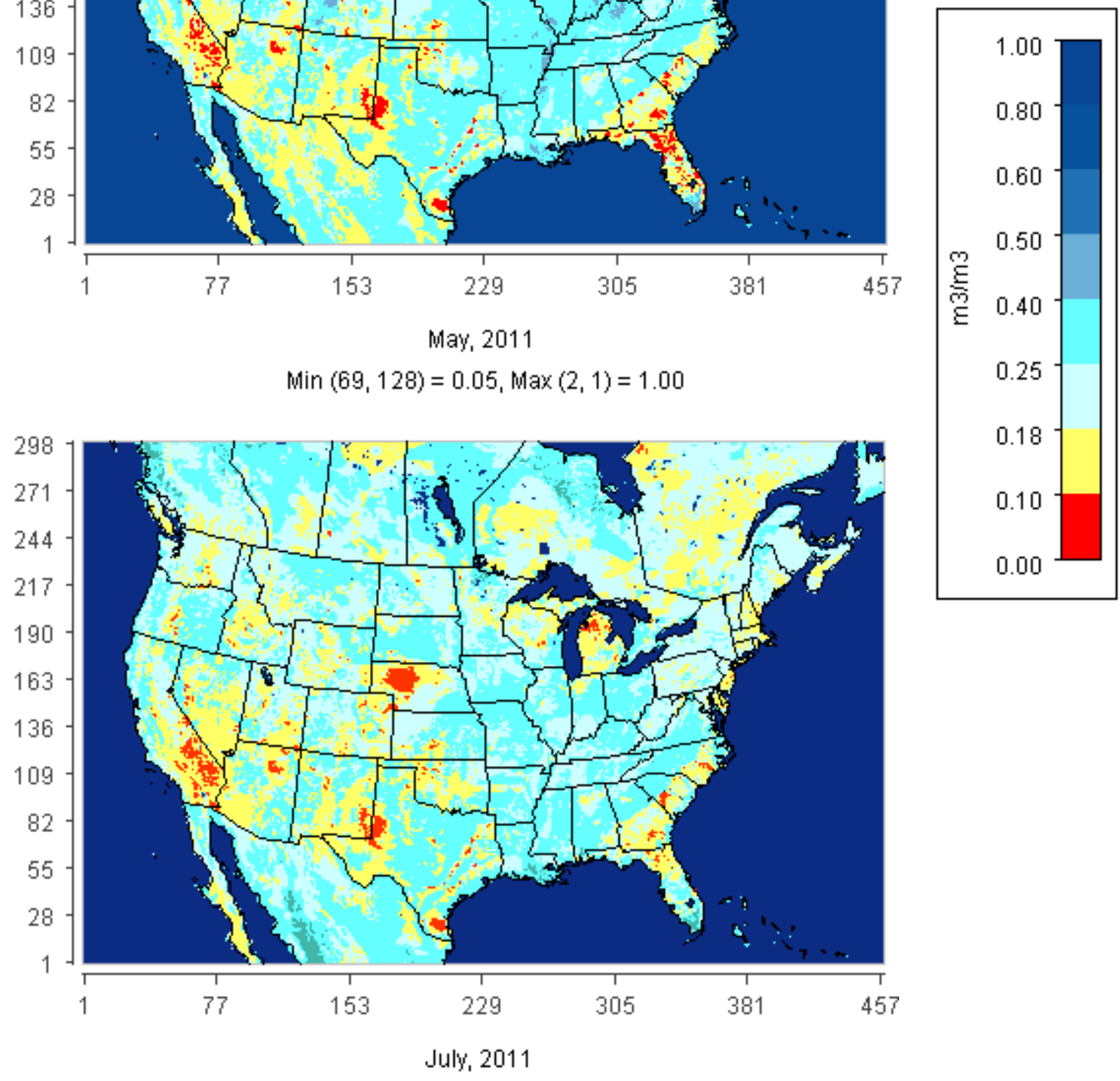

$\operatorname{Min}(201,72)=0.05, \operatorname{Max}(2,1)=1.00$ 